developed over food rewards. Rule-following mice in WBS trials displayed very little aggression, and the limited aggression observed had minimal impact on choice behaviour. Asymmetries in the sizes of the paired animals, which are a key determinant of social status, also had no effect on WBS-induced cooperation. Even when the authors reshuffled the mice into new pairs in which both animals had the same side preference (both monopolizing the left chamber in their previous trials, for instance), the animals rapidly re-established the alternate-side-allocation rule - thus demonstrating remarkable flexibility.

Choe and colleagues' experiments indicate that certain factors can put natural limitations on cooperation. These include food depriva$\operatorname{tion}^{8}$ and the presence of a powerful appetitive stimulus, the food pellet, which was clearly visible in the food-reward trials, and was presumably aromatic, too. By contrast, although WBS was associated with a light cue, it was otherwise not obvious to the unrewarded animal. These findings resonate with previous studies showing that the physical presence of tangible rewards impairs delayed gratification in blue jays ${ }^{9}$, complex rule-following by monkeys ${ }^{10}$ and chimps ${ }^{11}$, and cooperation in humans ${ }^{12}$.

The current study raises several questions. First, is social coordination by rule-following supported by the same neural circuit between the mediodorsal thalamus and dorsomedial prefrontal cortex that underlies status-based conflict resolution? If not, perhaps WBS overrides this circuit by triggering different circuits that stamp in a more 'cognitive' strategy.

Second, what role do internal states, such as hunger, have in strategy selection? The mice in Choe and colleagues' WBS trials were not food-deprived, and it would be interesting to determine how hunger would affect their behaviour.

And third, to what extent is rule-based coordination social at all? Determining to what extent this coordination depends on physical similarity between partners, transmission of social signals, or the implementation of a similar computational routine could provide clues to this question. If WBS could elicit the same type of coordination between a mouse and a robot, for example, this would demonstrate that the behaviour observed in the current study does not involve any sort of attribution of agency or strategic thinking, and instead arises from pure associative learning. Could WBS drive cooperation between the cartoon characters Tom the cat and Jerry the mouse, or might it just stop them from fighting?

Choe et al. have provided a compelling demonstration of a transition from aggressive to more-egalitarian interactions, at a time when examples of cooperation between animals in the laboratory are controversial and rare $^{8}$. Crucially, they have done so in mice, an animal model that will allow the whole range of powerful techniques in the neuroscience toolbox, from behaviour tracking to molecular-genetic tools such as optogenetics to electrophysiology, to be brought to bear on the these tricky but important social questions.

Scott M. Rennie is in the Champalimaud Neuroscience Programme, Champalimaud Centre for the Unknown, 1400-038 Lisbon, Portugal. Michael. L. Platt is in the Departments of Neuroscience, of Psychology and of Marketing, University of Pennsylvania, Philadelphia, Pennsylvania 19104, USA. e-mails:scott.rennie@neuro.fchampalimaud.org; mplatt@pennmedicine.upenn.edu
1. Hamilton, W. D. Am. Nat. 97, 354-356 (1963).

2. Trivers, R. L. O. Rev. Biol. 46, 35-57 (1971).

3. Stevens, J. R. \& Hauser, M. D. Trends Cogn. Sci. 8, 60-65 (2004)

4. Choe, I.-H. et al. Nature Commun. 8, 1176 (2017)

5. Wang, F., Kessels, H. W. \& Hu, H. Trends Neurosci. 37, 674-682 (2014)

6. Zhou, T. et al. Science 357, 162-168 (2017).

7. Olds, J. \& Milner, P. J. Comp. Physiol. Psychol. 47, 419-427 (1954).

8. Viana, D. S., Gordo, I., Sucena, E. \& Moita, M. A. P. PLOS ONE 5, e8483 (2010).

9. Stephens, D. W., McLinn, C. M. \& Stevens, J. R Science 298, 2216-2218 (2002).

10.Silberberg, A. \& Fujita, K. J. Exp. Anal. Behav. 66, 143-147 (1996).

11.Boysen, S. T. \& Berntson, G. G. J. Exp. Psychol. Anim. Behav. Processes 21, 82-86 (1995).

12.Nishi, A., Shirado, H., Rand, D. G. \& Christakis, N. A. Nature 526, 426-429 (2015).

This article was published online on 21 December 2017.

\title{
MICROBIOLOGY
}

\section{Pathogens boosted by food additive}

Epidemic strains of the bacterium Clostridium difficile have now been found to grow on unusually low levels of the food additive trehalose, providing a possible explanation for C. difficile outbreaks since 2001. SEE ARTICLE P.291

\section{JIMMY D. BALLARD}

B etween 2001 and 2006, epidemic strains of the bacterium Clostridium difficile, which can inhabit the bowel and cause dangerous diarrhoea, unexpectedly emerged in the United States, Canada and several European countries ${ }^{1,2}$. Most of these strains originated from a single lineage of $C$. difficile known as ribotype 027 (RT027; ref. 2), which has now spread around the world ${ }^{3}$. Of particular concern has been the correlation between RT027 and a dramatic increase in deaths related to $C$. difficile $e^{4}$. The mystery of why this ribotype and a second one, RT078, became so prevalent apparently out of thin air has remained largely unsolved ${ }^{5}$. On page 291, Collins et al. ${ }^{6}$ raise the possibility that the seemingly harmless addition of a sugar called trehalose to the food supply contributed to this disease epidemic.

Collins and colleagues first explored how RT027 and RT078 grow, by comparing carbon-source preferences between strains of C. difficile. They noted a peculiar property of these two lineages - they can use low concentrations of trehalose as a sole source of carbon. Next, the authors analysed the genomes of RT027 and RT078, and discovered that each encodes unusual sequences that might explain their ability to grow in low levels of trehalose.

The researchers showed that RT027 carries a single-nucleotide genetic variant that changes an amino-acid residue in the protein TreR from leucine to isoleucine. Tre $\mathrm{R}$ is a transcriptional repressor that is inhibited by trehalose. When active, TreR prevents expression of the gene tre $A$, which encodes a phosphotrehalase enzyme involved in metabolizing trehalose into glucose and glucose derivatives. Thus, trehalose is metabolized only when its levels are high enough to inhibit TreR. Collins et al. propose that the mutation in RT027 changes TreR's affinity for trehalose and allows it to be repressed by substantially lower levels of the sugar than normal. This frees the TreA protein to metabolize trehalose and allows RT027 to grow on low levels of the sugar (Fig. 1).

By contrast, RT078 has adapted to grow on low amounts of trehalose by acquiring four genes involved in trehalose uptake and metabolism. The genes encode second copies of TreR and TreA, a trehalose transporter protein dubbed PtsT that helps cells take up the sugar, and another enzyme, TreX, involved in trehalose metabolism. Unexpectedly, RT078 does not share the genetic alteration in TreR that is found in RT027. As Collins and colleagues point out, it therefore seems that two epidemic strains of C. difficile have optimized trehalose metabolism in unrelated ways.

The investigators next provided evidence that trehalose metabolism directly relates to enhanced virulence of RT027 in vivo. First, they showed that deleting treA in RT027 and thereby preventing trehalose metabolism 


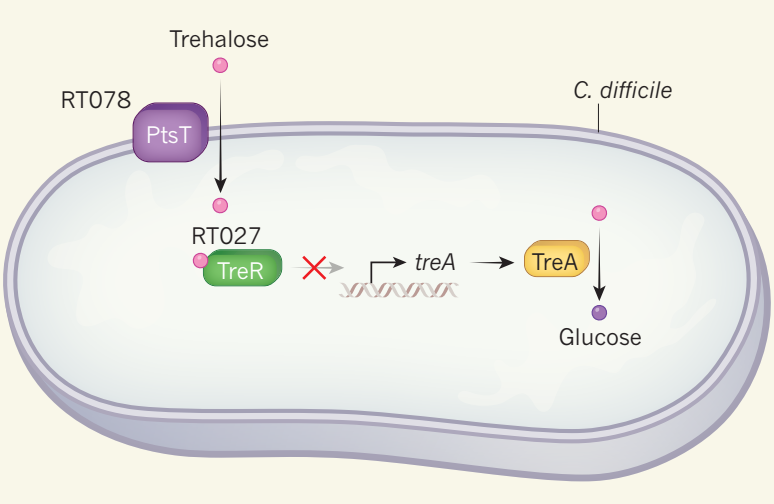

Figure 1 | Increased virulence of the bacterium Clostridium difficile. Two lineages of C. difficile, RT027 and RT078, have become widespread since the early 2000s. Collins et al. ${ }^{6}$ have demonstrated that different mutations have arisen in each strain to improve the microbes' ability to grow on low concentrations of the sugar trehalose, which has been added to foods since 2001. RT078 has acquired four genes, including one that encodes the protein PtsT, which transports trehalose into C. difficile cells. In RT027, mutation of the protein TreR increases the protein's affinity for trehalose, which in turn inhibits TreR's ability to bind to DNA and repress transcription of the gene treA. TreA protein, expressed when TreR is repressed, metabolizes trehalose to glucose and derivatives, enabling cell growth at low trehalose concentrations.

markedly reduced the virulence of this strain in mice. Second, adding trehalose to the diet of mice infected with RT027 increased the animals' risk of death. However, the bacterial load of RT027 was not higher in mice fed trehalose than in those on a trehalose-free diet, indicating that increased risk of death is not simply due to the presence of more bacteria. Rather, the authors found that improved trehalose metabolism enables RT027 to produce higher levels of a $C$. difficile toxin.

Turning to RT078, Collins et al. demonstrated that just one of the four acquired proteins, the trehalose transporter PtsT, was responsible for the strain's increased ability to grow on low levels of trehalose (Fig. 1). The authors showed that PtsT confers a competitive growth advantage over other lineages in the presence of trehalose.

Finally, Collins and colleagues investigated the relevance of their observations in humans. Experimental infection would be difficult in people, so the researchers instead collected fluid from the small intestine of three participants on a normal diet. The fluid contained levels of trehalose sufficient to promote expression of treA in RT027 but not in other strains, supporting the potential for human relevance.

RT027 was first isolated in 1985, from a person infected with $C$. difficile. But this ribotype was not associated with hospital outbreaks, increased death rates or epidemics until the early 2000s. Similarly, RT078 lineages isolated before the C. difficile epidemics carry the genetic information for enhanced trehalose metabolism, but were of little consequence to the epidemiology of this disease. Why did these ribotypes suddenly emerge at epidemic levels only 15 years ago?

Collins and colleagues propose a surprising answer. Before 1995, high production costs made trehalose untenable as a food additive. But manufacturing innovations ${ }^{7}$ reduced the cost of trehalose production more than $100-$ fold $^{8}$, and the US Food and Drug Administration and European agencies approved the sugar as a safe food additive in 2000 and 2001, respectively (see go.nature.com/2yewlwk; go.nature.com/2jyltr3). Trehalose is now added to a variety of food products, including pasta, ice cream and minced beef. The authors provide a timeline (see Fig. 6 of the paper) to illustrate how supplementing the food supply with trehalose preceded the $C$. difficile outbreaks caused by RT027 and RT078. They therefore suggest that the addition of trehalose to the food supply might have increased

\section{SUSRUTA MAJUMDAR \& LAKSHMI A. DEVI}

$\mathrm{E}$ ffective pain management is one of the greatest challenges of modern medicine. Opioids such as morphine and fentanyl are the preferred clinical treatments for moderate to severe pain because of their strong analgesic (pain-relieving) effects. But the ongoing epidemic of deaths from respiratory the sugar in the human bowel to levels high enough to enable growth of these ribotypes.

The study's findings raise several avenues for future research. For instance, the connection between trehalose metabolism and toxin production, and how this is linked to increased death rates in people infected with RT027, will require further analysis. Whether trehalose in the human colon, where disease occurs, reaches high enough levels to affect RT027 and RT078 virulence is also unknown. The authors tested fluid from the small intestine, thus bypassing the colon, where the complex complement of gut microbes might break down trehalose.

Despite these concerns, the correlative findings of Collins and colleagues' study are compelling. It is impossible to know all the details of events surrounding the recent $C$. difficile epidemics, but the circumstantial and experimental evidence points to trehalose as an unexpected culprit.

Jimmy D. Ballard is in the Department of Microbiology and Immunology, University of Oklahoma Health Sciences Center, Oklahoma City, Oklahoma 73190, USA.

e-mail:jimmy-ballard@ouhsc.edu

1. Bartlett, J. G. Ann. Intern. Med. 145, 758-764 (2006).

2. McDonald, L. C. et al. N. Engl. J. Med. 353, 2433-2441 (2005).

3. He, M. et al. Nature Genet. 45, 109-113 (2013).

4. Hunt, J. J. \& Ballard, J. D. Microbiol. Mol. Biol. Rev. 77, 567-581 (2013).

5. Kuijper, E. J., van Dissel, J. T. \& Wilcox, M. H. Curr. Opin. Infect. Dis. 20, 376-383 (2007).

6. Collins, J. et al. Nature 553, 291-294 (2018).

7. Maruta, K. et al. Biosci. Biotechnol. Biochem. 59, 1829-1834 (1995).

8. Higashiyama, T. Pure Appl. Chem. 74, 1263-1269 (2002).

This article was published online on 3 January 2018.

\section{Strategy for making safer opioids bolstered}

Compounds have been made that activate only the G-protein signalling pathway when bound to the $\mu$-opioid receptor - the target of opioid pain relievers. These compounds lack one of the main side effects of currently used opioids.

depression induced by opioid overdoses highlights the need for safer analgesics. Writing in Cell, Schmid et al. ${ }^{1}$ report a series of compounds that provides a much-needed proof of principle of a strategy for making safer opioids.

It is estimated that more than 100,000 adults suffer from chronic pain in the United States alone, and that this costs up to US\$635 billion per year in medical treatment and lost 